\title{
Interference and Clock Drift Effects in UWB RFID Systems Using Backscatter Modulation
}

\author{
Nicolò Decarli* Francesco Guidi* ${ }^{* \dagger}$, Andrea Conti ${ }^{\ddagger}$, and Davide Dardari* \\ ${ }^{*}$ CNIT, Wilab, University of Bologna, via Venezia 52, 47023 Cesena (FC), Italy \\ email: \{f.guidi, nicolo.decarli, davide.dardari\}@unibo.it \\ ${ }^{\dagger}$ ENSTA-ParisTech, 32 Boulevard Victor, 75739 Paris Cedex 15, France \\ email: francesco.guidi@ensta-paristech.fr

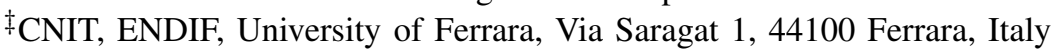 \\ email: a.conti@ieee.org
}

\begin{abstract}
UWB backscatter modulation paves the way to next generation RFID systems. This solution overcomes the main limitations of the current UHF technology in terms of robustness to multipath fading and localization capability. On the other hand, the design of UWB RFID systems presents several new aspects to deal with such as clutter, multi-user interference, and clock drift. This paper illustrates some solutions able to guarantee reliable communication using semi-passive tags jointly with UWB backscatter modulation in the presence of implementation impairments.
\end{abstract}

Index Terms-backscatter, clock drift, interference, RFID, UWB

\section{INTRODUCTION}

There is a growing interest in the convergence of radiofrequency identification (RFID) and high-definition real time locating systems (RTLS) technologies to enhance the functionalities offered to the end user and enable new potential wide market applications [1]-[3]. ${ }^{1}$ Ultrawide bandwidth (UWB) technology leads to advantages in terms of communication robustness, localization accuracy, multi-tag capability, and interference rejection, even in harsh propagation environments [4], [5]. When extremely low-power consumption tags are required semi-passive tags represent one of the most promising solution. In semi-passive tags using backscatter modulation, the energy available from harvesting or batteries is used only for memory access or modulation operation without powering an active transmitter. The high accuracy estimation of the timeof-arrival (TOA) from the backscatter signals enables accurate localization of tags in addition to their detection [2], [6]-[9].

Figure 1 shows an example of a scenario (cell) composed of 4 readers in a square room, where each reader interrogates $N_{\text {tag }}$ tags located in the same area. To save energy, tags are normally in sleep state and are woken up through the transmission of a wake-up signal (e.g., an unmodulated UHF carrier). Then all tags reflect the incoming UWB signals by means of backscatter modulation according to their internal

\footnotetext{
${ }^{1}$ This is one of the objectives of the European project SELECT which focuses on studying new solutions for enabling high-accuracy detection, identification, and location of objects/persons equipped with small ultralow power tags using a network of intelligent self-configuring radio devices (readers). http://www. selectwireless.eu
}

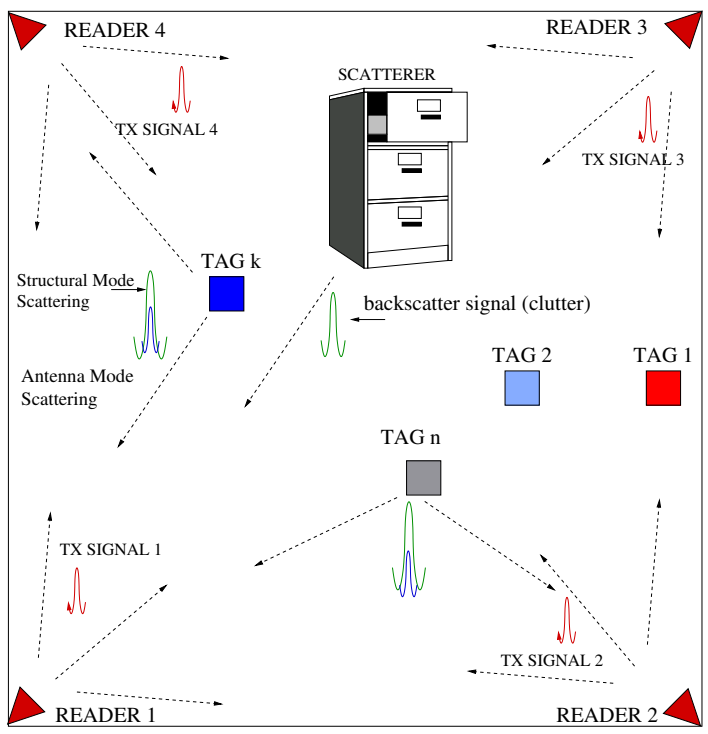

Fig. 1. Multi-reader scenario with $N_{\text {tag }}$ tags.

information bit. As will be detailed in the next section, signals backscattered by different tags and originated by different readers can be distinguished through spreading codes at both reader and tag sides. Several issues arise due to the presence of clutter (the signal backscattered by the environment), multi-tag interference, tag clock drift, and the poor link budget for to the two-hop link. These issues have been partially and separately investigated in the literature [10]-[13].

In this paper, we present a system architecture for mitigating the presence of multi-tag interference and of strong clock drift. A low complexity non coherent detection scheme is analyzed and spreading code design strategies are investigated. Finally, simulative results assess the performance in terms of detection rate as a function of the false alarm rate.

The reminder of the paper is organized as follows. Section II presents the considered tag-reader communication system. The proposed low-complexity detection scheme is described in Section III. In Section IV, an analysis of the code families suitable for the investigated RFID application is provided. 


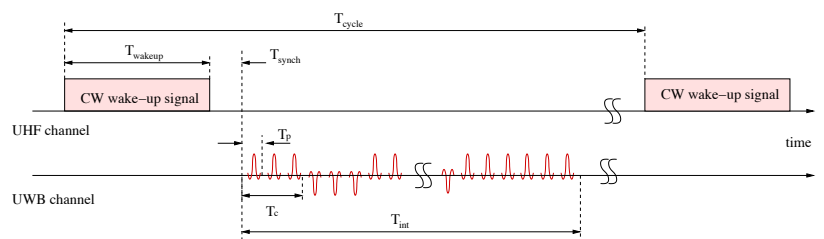

Fig. 2. The reader interrogation signal structure in the UHF and UWB bands.

The system performance is analyzed in Section V. Finally, conclusions are given in Section VI.

\section{TAG-ReAder COMMUNiCATION}

We consider a tag-reader communication scheme based on UWB backscatter modulation where each reader is composed of a transmitter and a receiver section that are connected to an UWB antenna [10], [13].

During the interrogation cycle of duration $T_{\text {cycle }}$ (see Fig. 2), the reader sends a wake-up carrier in the UHF bands of duration $T_{\text {wakeup }}$ followed, after a fixed time $T_{\text {synch }}$, by a sequence of UWB pulses of duration $T_{\mathrm{int}}$. These pulses are modulated by a periodic antipodal binary spreading sequence $\left\{d_{n}\right\}$ of period $T_{\mathrm{s}}$ and length $N_{\mathrm{c}}$ chips specific for each reader (reader's code). Each code chip is composed of $N_{\mathrm{pc}}$ pulses, therefore to accommodate the signal backscattered by tags corresponding to an entire packet of $N_{\mathrm{r}}$ bits, the UWB interrogation consists of $N_{\mathrm{t}}=N_{\mathrm{r}} N_{\mathrm{s}}$ pulses, where $N_{\mathrm{s}}=N_{\mathrm{c}} N_{\mathrm{pc}}$. Considering a pulse repetition period (PRP) of $T_{\mathrm{p}}$ seconds, the packet duration results in $T_{\mathrm{int}}=N_{\mathrm{r}} T_{\mathrm{s}}=N_{\mathrm{r}} N_{\mathrm{s}} T_{\mathrm{p}}$. In a single packet, the transmitted UWB interrogation signal is given by

$$
s_{\text {reader }}(t)=\sum_{k=0}^{N_{\mathrm{r}}-1} s\left(t-k N_{\mathrm{c}} T_{\mathrm{c}}\right)
$$

where $s(t)=\sum_{n=0}^{N_{\mathrm{c}}-1} d_{n} g\left(t-n T_{\mathrm{c}}\right)$ and $g(t)=\sum_{i=0}^{N_{\mathrm{pc}}-1} p(t-$ $\left.i T_{\mathrm{p}}\right)$ is the waveform associated to each chip composed of $N_{\mathrm{pc}}$ elementary UWB pulses. The PRP $T_{\mathrm{p}}$ is chosen so that all backscattered signals are received by the reader before the transmission of the successive pulse, thus avoiding interframe interference. Each pulse in (1) is backscattered by all tags as well as by all the surrounding scatterers present in the environment (clutter component). Figure 3 shows the structure of the tag where antenna backscattering properties are changed by varying the antenna load using an UWB switch (open and short circuit conditions). ${ }^{2}$ To make the uplink communication between the $k$ th tag and the reader robust to the presence of clutter and interference as well as to allow multiple access, each tag is designed to change its status (short or open circuit) at each chip time $T_{\mathrm{c}}=N_{\mathrm{pc}} T_{\mathrm{p}}$, according to the data to be transmitted and an antipodal tag's code $\left\{c_{n}^{(k)}\right\}$ of period $N_{\mathrm{c}}$. Specifically, each antipodal tag information symbol $b_{n}^{(k)}$ is associated to $N_{\mathrm{s}}$ pulses, with symbol time $T_{\mathrm{s}}=T_{\mathrm{p}} N_{\mathrm{s}}$. In this way the polarity of the reflected signal changes each

\footnotetext{
${ }^{2}$ In [10] it is shown that this affects the polarity of the backscattered antenna mode component.
}

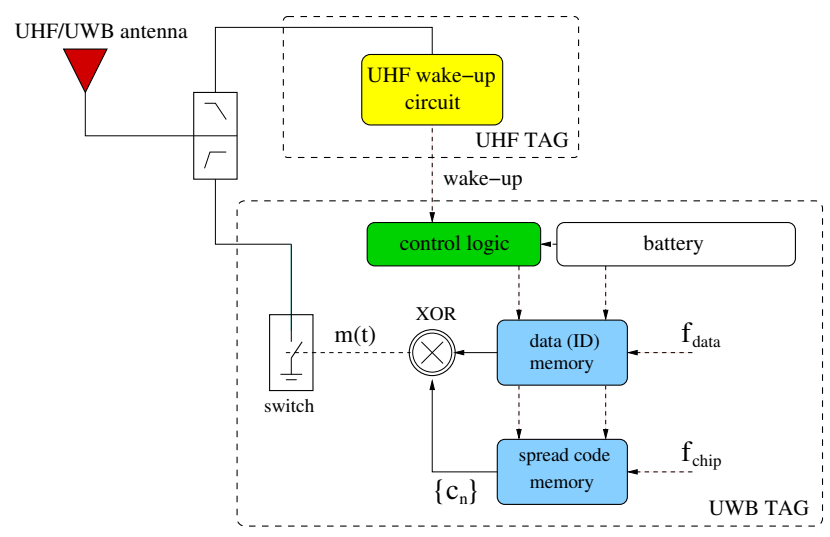

Fig. 3. Tag internal structure.

chip time according to the tag's code sequence, whereas the information symbol affects the entire sequence pulse polarity at each symbol time. The reader and the tags have independent clock sources, thus they are asynchronous. However, the wakeup signal can also be exploited to reset the tag spreading code generator as shown in Fig. 3. This allows considering the system as quasi-synchronous, thus facilitating code design and drastically reducing the code acquisition time as will be clarified afterward. The presence of a low cost oscillator in the tag and the long duration of the symbol (a typical value of $T_{\mathrm{s}}$ is about $1 \mathrm{~ms}^{3}$ ) make the clock drift effect not negligible after the reception of a few bits. In particular, the clock skew between the $k$ th tag and the reader can be modeled as $\delta^{(k)}(t)=T_{\mathrm{o}}^{(k)}+D^{(k)} t$, where $T_{\mathrm{o}}^{(k)}$ is the residual initial offset after the wake-up synchronization process, and $D^{(k)}$ is the clock drift. Practical worst case values for $T_{0}^{(k)}$ and $D^{(k)}$ are, respectively, $\pm 500 \mathrm{~ns}$ and $\pm 100 \mathrm{ppm}$. Therefore, the backscatter modulator signal, commanding the tag switch is given by

$$
\begin{aligned}
m^{(k)}(t) & =\sum_{n=0}^{N_{\mathrm{r}}-1} \sum_{i=0}^{N_{\mathrm{c}}-1} c_{i}^{(k)} b_{n}^{(k)} \\
& \cdot \Pi\left(\frac{1}{\widetilde{T}_{\mathrm{c}}}\left[t-n N_{\mathrm{c}} \widetilde{T}_{\mathrm{c}}^{(k)}-i \widetilde{T}_{\mathrm{c}}^{(k)}-T_{\mathrm{o}}^{(k)}\right]\right)
\end{aligned}
$$

with $\widetilde{T}_{\mathrm{c}}^{(k)}=T_{\mathrm{c}}\left(1+D^{(k)}\right)$ and $\Pi(t / \tau)$ the rectangular function of duration $\tau$. The signal received by the reader is ${ }^{4}$

$$
\begin{aligned}
r_{\text {reader }}(t)= & \sum_{k=1}^{N_{\text {tag }}}\left[\left(s_{\text {reader }}(t) \otimes h^{(k)}(t)\right) \times m^{(k)}(t)\right] \otimes h^{(k)}(t) \\
& +s_{\text {reader }}(t) \otimes h^{(\mathrm{C})}(t)+n(t)
\end{aligned}
$$

where $h^{(k)}(t)$ is the channel impulse response related to the reader- $k$ th tag link, $h^{(\mathrm{C})}(t)$ is the impulse response of the environment for the clutter, and $n(t)$ is the additive white gaussian noise (AWGN) with two-sided power spectral density $N_{0} / 2$. The tag antenna structural mode is treated as a part

\footnotetext{
${ }^{3}$ This and the next system specifications are driven by the outcome of the European project SELECT, http://www.selectwireless.eu

${ }^{4}$ Operator $\otimes$ denotes the convolution.
} 
of clutter since it does not depend on data symbols. Note that signals backscattered by tags are received through double convolution with the channel impulse response.

\section{TAG DETECTION}

The estimation of the two-way backscatter channel is more difficult than in conventional one-way channels, then the adoption of non coherent schemes based on energy detectors is preferable. In the following we propose a non coherent approach which consists in a series of de-spreading operations followed by energy evaluations. Specifically, de-spreading is performed based on a sequence that is obtained as product of the spreading code $\left\{d_{n}\right\}$ related to the reader and a shifted version of the spreading code $\left\{c_{n}\right\}$ related to the intended useful tag, discriminating the backscatter signal associated to a specific reader-tag couple. We define the periodically repeated sequences (with period $\left.N_{\mathrm{s}}\right) \tilde{c}_{m} \triangleq c_{\left\lfloor m / N_{\mathrm{pc}}\right\rfloor}$ and $\tilde{d}_{m} \triangleq d_{\left\lfloor m / N_{\mathrm{pc}}\right\rfloor \sim}$, for $m=0,1, \ldots N_{\mathrm{s}}-1$, with $\tilde{c}_{m+N_{\mathrm{s}}}=\tilde{c}_{m}$ and $\tilde{d}_{m+N_{\mathrm{s}}}=\tilde{d}_{m}$ for any $m$.

The wake-up synchronization offset $T_{\mathrm{o}}^{(k)}$ and the clock drift $D^{(k)}$ generate an uncertainty on the offset (phase) of the tag spreading code with respect to the reader local clock. Note that, contrarily to conventional active transmission schemes, the TOA (hence the PRP) of the received pulses is not affected by the clock drift signals generated by the same reader. Only the way they are modulated depends on these effects. Therefore, the code acquisition and detection schemes require the availability of de-spreading outputs for different code shifts within the expected maximum variation. This is achieved by correlating the samples with differently shifted versions of $\tilde{c}_{m}$ and in-phase version of $\tilde{d}_{m}$. In particular, $N_{\text {span }}$ shifts with span step $\Delta$ are considered. The values of $N_{\text {span }}$ and $\Delta$ have to be determined according to the robustness of codes with respect to shifts and to the entity of clock drift and initial offset. Consequently the acquisition window is $\Delta\left(N_{\text {span }}-1\right) T_{\mathrm{p}}$ seconds, where $N_{\text {span }}$ is considered to be odd. In addition, $N_{\text {span }}$ cannot be too large to keep the receiver complexity affordable and to prevent high probability of false alarm (PFA). Without loss of generality, we consider the detection of tag 1 by observing only the first bit of the packet i.e., $n=0$ in (2). Tag detection is based on the construction of a $N_{\text {span }} \times N_{\text {bin }}$ energy matrix $\mathbf{E}=\left\{e_{n, m}\right\}$, with $N_{\text {bin }}=\left\lfloor T_{\mathrm{p}} / T_{\mathrm{ED}}\right\rfloor$ representing the number of integration bins each PRP is divided in, having denoted with $T_{\mathrm{ED}}$ the integration time. ${ }^{5}$ Energy samples are related to the energy observed in different time bins of duration $T_{\mathrm{ED}}$ after the despreading process as given by

$$
e_{n, m}=\int_{(m-1) T_{\mathrm{ED}}}^{m T_{\mathrm{ED}}}\left[\sum_{l=0}^{N_{\mathrm{s}}-1} \tilde{d}_{l} \tilde{c}_{l+(n+\nu) \Delta}^{(1)} r_{\text {reader }}\left(t-l T_{\mathrm{p}}\right)\right]^{2} d t
$$

with $m=1,2, \ldots, N_{\text {bin }}, n=1,2, \ldots, N_{\text {span }}$, and $\nu=$ $-\left(N_{\text {span }}+1\right) / 2$.

The simplest strategy for tag detection is to locate the maximum element of the energy matrix $\mathbf{E}$. If the value is above a

\footnotetext{
${ }^{5}$ This time is on the order of the pulse duration (i.e., few ns).
}

certain threshold $\xi$, then the target is detected. The coordinates $(\widehat{n}, \widehat{m})$ associated to the maximum provide, respectively, an estimate of the tag clock offset and a coarse estimate of the signal TOA, which can be improved by adopting ranging strategies as in [7]. The choice of the threshold affects the performance of the detection scheme in terms of probability of detection (PD) and PFA. Low values for the threshold lead to higher PFA and higher PD. The vice versa holds for high values of $\xi$ (low PFA but low PD). Note that, while the max search strategy is a good option in AWGN channels and in the absence of interference, it is not the optimal one in the presence of multipath propagation and interference.

\section{TAG CODE DESIGN}

The system performance strongly depends on spreading code design that must satisfy several requirements in terms of number of available sequences, robustness to strong clock drift and wake-up offset, multi-user interference mitigation, and clutter removal capability. In addition, during the reception of the first bits of the packet, the system is quasi-synchronous since all tags are woken up simultaneously within a maximum initial wake up offset in the order of $T_{\mathrm{c}}$, but after a few bits it tends to become asynchronous when strong tag clock drift is present. For what clutter removal capability is concerned, in [13] it has been shown that it can be achieved by adopting balanced codes (i.e., codes with the same number of +1 and -1 in each codeword). The robustness to clock offset can be obtained with a code family that can guarantee the orthogonality (or very small peak cross correlation) even when small shifts are present. Zero correlation zone codes [14] allow to extend the orthogonality among codewords for a certain number $Z$ of shifts. Then, the interference is canceled also in presence of synchronization offset. On the other hand, low correlation zone codes mitigate the interference, without totally canceling it, for a certain number $L$ of shifts. Many codes of this type have been proposed for quasi-synchronous code division multiple access (CDMA) systems, with different performance in terms of $L / Z$ zone length, maximum cross-correlation and number of available codewords [15]. Another simple approach is to increase $N_{\text {pc }}$ through simple chip duplication. While this gives a lower number of codewords, potential lower interference mitigation properties, and lower clutter rejection if an odd code is adopted, it enhances robustness to codes shifts.

Orthogonal codes, such as Walsh Hadamard, offer optimal interference rejection in synchronous systems, but in asynchronous conditions they present unsatisfactory crosscorrelation properties [16]. Gold and Kasami pseudo-noise (PN) codes are good candidates in this case, but they are not balanced and usually do not behave as well as Orthogonal codes in synchronous scenario [16]. An interesting possibility for the UWB RFID backscatter modulation system is to adopt orthogonal Gold codes [17]. These codes are exactly orthogonal in the synchronous scenario, as they are phasealigned to assure orthogonality, and maintains the properties of extended Gold Codes (low cross-correlation) in asynchronous scenario. They are constructed by the lengthening of one chip 


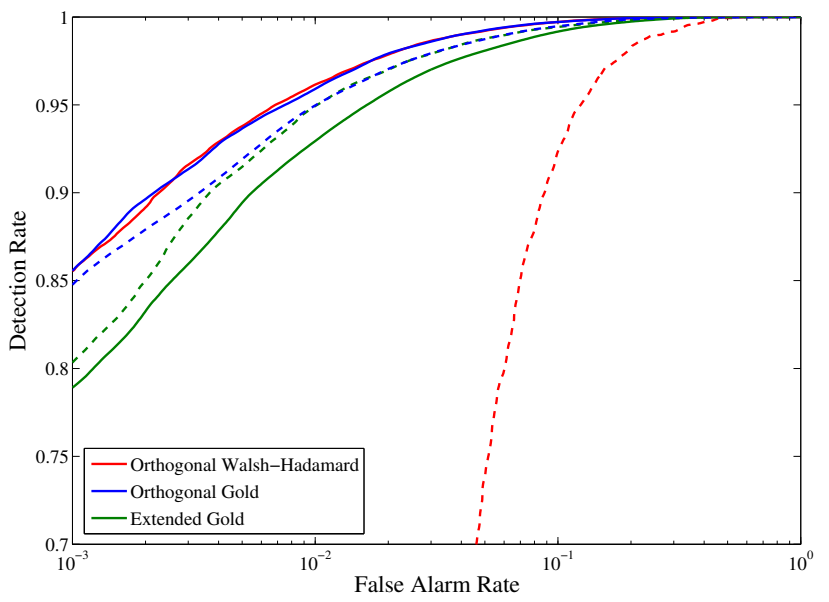

Fig. 4. ROC for the tag detection in UWB backscatter system. Continuous lines (-) refer to the perfectly synchronous scenario, dashed lines (- -) refer to the asynchronous scenario due the presence of clock drift, with ideal code phase retrieving.

preferentially-phased Gold codes [18], where a code phase optimization process is performed in order to assure proper cross-correlation properties [17]. Therefore, an even code is obtained, which enables the complete clutter cancellation and good interference rejection for various scenarios. The possibility of adopting such a family of codes is very interesting in presence of a wake-up synchronization offset and drift. In the numerical results the impact of different coding strategies on the detection performance will be investigated.

\section{NUMERICAL RESUlTS}

We now analyze the tag detection rate as a function of the false alarm rate considering the detection strategy based on energy measurements proposed in Sec. III. A total number of pulses per symbol $N_{\mathrm{s}}=8192$ is chosen, ${ }^{6}$ in order to counteract the poor link budget, with $T_{\mathrm{c}}=1.024 \mu \mathrm{s}$ and $T_{\mathrm{p}}=128 \mathrm{~ns}$ (i.e., $N_{\mathrm{pc}}=8$, which means $N_{\mathrm{c}}=1024$ ). A reader with $5 \mathrm{dBi}$ antenna gain, a tag considered in the maximum reader's antenna direction equipped with an $1 \mathrm{dBi}$ antenna and $2 \mathrm{~dB}$ switch losses have been considered. Results have been obtained by Monte Carlo simulations, starting from multipath channel responses with exponential power delay profile and Nakagami- $m$ fading (severity factor $m=3$ ), a root-meansquared channel delay-spread of $10 \mathrm{~ns}^{7}$ and paths separated of $2 \mathrm{~ns}$ apart. A transmitted signal compliant with the IEEE 802.15.4a emission mask in the $3-5 \mathrm{GHz}$ is considered. For what concerns the clutter, a worst-case of uniform power delay profile in the overall interval $T_{\mathrm{p}}$ is considered, with paths spaced $0.95 \mathrm{~ns}$ apart, each path with amplitude characterized by Nakagami- $m$ fading, with $m=3$, and a root-meansquared value of $0.5 \mathrm{mV}$ at the receiver. The non-coherent

\footnotetext{
${ }^{6}$ This value derives from the number of pulses necessary to achieve a certain signal-to-noise ratio (SNR) per bit given the maximum reader-tag reading range (link budget constraint).

${ }^{7}$ This value is comprehensive of the two-way link of the backscatter signal.
}

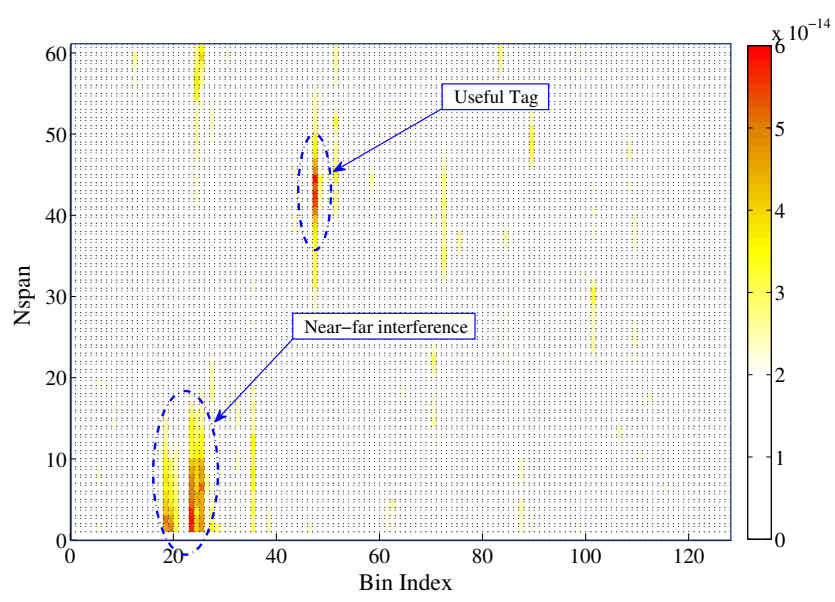

Fig. 5. Example of energy matrix $\mathbf{E}$ in presence of synchronization offset, clock drift and near-far interference effects.

low-complexity detector implementation of Sec. III is adopted, considering an energy detection interval $T_{\mathrm{ED}}=1 \mathrm{~ns}$.

In Fig. 4, we report the receiver operating characteristics (ROC) corresponding to perfectly synchronous and asynchronous scenarios with ideal code phase retrieving, related to a useful tag placed at $7 \mathrm{~m}$ from the reader. We consider 59 interfering tags uniformly distributed in two meters around the useful tag, to reproduce interference effects. In particular, orthogonal Walsh codes in synchronous scenario represent the benchmark, since the interference is completely removed. On the contrary, their performance drastically degrades when the scenario becomes asynchronous. For what extended Gold codes are concerned, obtained by lengthening of one chip the Gold codes without any kind of phase optimization when aligned, they evidence a loss while the scenario is synchronous, but they allow satisfactory detection capabilities also in asynchronous conditions. Orthogonal Gold codes represent instead the best trade-off for both scenarios since they achieve a detection rate higher than 0.8 with a false alarm of $10^{-3}$ also in the asynchronous scenario, while maintaining the optimal behavior of Walsh codes in perfectly synchronous scenario.

It should be remarked that in this simulation near-far effects are negligible, as all the users were approximately placed at the same distance from the reader. In a real scenario, it may happen to have some interfering tags significantly closer than the useful one to the reader, with the consequence of potential near-far effects. In this case classical power control approaches of CDMA systems cannot be adopted due to the passive communication nature of the system. As example, Fig. 5 shows the energy matrix $\mathbf{E}$ for a case study where the path loss difference between the useful tag and interference tags closer to the reader is about $20 \mathrm{~dB}$. If a constant threshold over all the bins were adopted, the probability of false alarm due to an interferer very close to the reader would increase significantly. This effect is particularly empathized since in UWB backscattering systems the path loss difference for tags at different distances from the reader (i.e., the dynamic range 


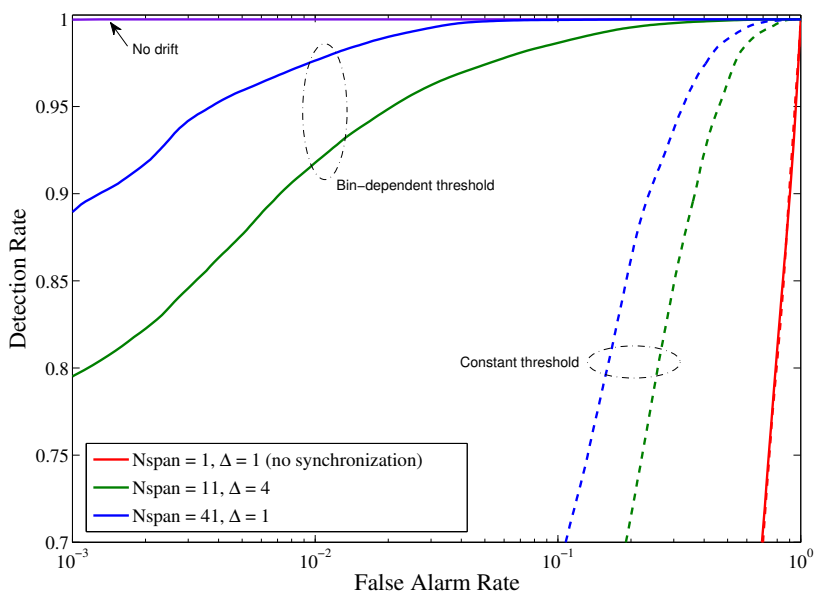

Fig. 6. ROC for tag detection in presence of synchronization offset, clock drift and near-far interference effects. Continuous lines (-) refer to the bindependent threshold, dashed lines (- -) refer to the constant threshold.

at the receiver excluding the clutter effect) could be significant due to the two-hop link. To understand this problem, Fig. 6 shows the ROC when the useful tag is placed at $6 \mathrm{~m}$ from the reader to guarantee a detection rate of about 0.9 with false alarm of $10^{-3}$ even with synchronization losses, and 19 interfering tags are uniformly distributed between $2.8 \mathrm{~m}$ and $3.2 \mathrm{~m}$. Orthogonal Gold codes are adopted, a synchronization offset of $500 \mathrm{~ns}$, and a drift of $100 \mathrm{ppm}$ are considered. The 16 th bit of the transmitted preamble (a sequence of all ' +1 ') is analyzed for detection purposes and the acquisition window is fixed to $40 T_{\mathrm{p}}$ when performing synchronization. As clearly depicted in Fig. 6, the system suffers of near-far effects when a constant threshold over all the bins is considered (dashed lines). Thus a possible solution is represented by the adoption of a threshold which accounts for the interference effects, that is, a bin-dependent variable threshold $\xi_{m}, m=1,2, \ldots, N_{\text {bin }}$, computed such that, in the presence of interference, the PFA is constant for each bin. An empirical approach adopted for simulations considers a threshold reflecting the tag path-loss behavior until the tag energy, evaluated in $T_{\mathrm{ED}}$, falls below the noise floor $N_{0} W T_{\mathrm{ED}}$, then fixing the threshold to a constant value for the last bins. Figure 6 shows that, when the bindependent threshold is adopted (continuous lines), near-far effects are significantly mitigated and a detection rate higher than 0.8 with a false alarm of $10^{-3}$ can be achieved when $N_{\text {span }}$ is set to 41 to counteract the clock offset. Vice-versa, adopting $N_{\text {span }}=11$, a lower detection rate is obtained since the synchronization scheme is less robust for the considered $N_{\mathrm{c}}=1024$. Fixing $N_{\text {span }}=1$, i.e., without code synchronization, no detection capabilities can be assured even when a bin-dependent threshold is chosen.

\section{CONCLUSION}

In this paper we presented UWB RFID systems based on backscatter modulation, in presence of interference, wake-up synchronization offset and clock drift. Tag detection performance has been evaluated in terms of detection and false alarm rate, to analyze the robustness of different code families in different scenarios. It has been shown that the joint use of orthogonal Gold codes with proper low-complexity detection and synchronization schemes involving bin-dependent thresholding is a promising solution to overcome implementation impairments and near-far effects.

\section{ACKNOWLEDGMENT}

This work has been funded by the European Commission through the FP7 project SELECT (grant agreement n 257544). Authors would like to thank Marco Chiani and Stefania Bartoletti for fruitful discussions.

\section{REFERENCES}

[1] D. Dardari, E. Falletti, and M. Luise, in Satellite and Terrestrial Radio Positioning Techniques - A signal processing perspective. Elsevier Ltd, London, 2011.

[2] D. Dardari, R. D’Errico, C. Roblin, A. Sibille, and M. Z. Win, "Ultrawide bandwidth RFID: The next generation?" Proc. of the IEEE, vol. 98 , no. 9 , pp. $1570-1582$, Sep. 2010.

[3] M. Win, A. Conti, S. Mazuelas, Y. Shen, W. Gifford, D. Dardari, and M. Chiani, "Network localization and navigation via cooperation," IEEE Communications Magazine, vol. 49, no. 5, pp. 56-62, May 2011.

[4] M. Win and R. Scholtz, "Impulse radio: how it works," IEEE Communications Letters, vol. 2, no. 2, pp. 36-38, Feb. 1998.

[5] — , "Ultra-wide bandwidth time-hopping spread-spectrum impulse radio for wireless multiple-access communications," IEEE Transactions on Communications, vol. 48, no. 4, pp. 679-689, Apr. 2000.

[6] Y. Shen and M. Win, "Fundamental limits of wideband localization; part I: A general framework," IEEE Transactions on Information Theory, vol. 56, no. 10, pp. 4956-4980, Oct. 2010.

[7] D. Dardari, A. Conti, U. Ferner, A. Giorgetti, and M. Z. Win, "Ranging with ultrawide bandwidth signals in multipath environments," Proc. of IEEE, vol. 97, no. 2, pp. 404-426, Feb. 2009.

[8] S. Gezici, Z. Tian, G. B. Giannakis, H. Kobayashi, A. F. Molisch, H. V. Poor, and Z. Sahinoglu, "Localization via ultra-wideband radios: a look at positioning aspects for future sensor networks," IEEE Signal Processing Mag., vol. 22, pp. 70-84, Jul. 2005.

[9] A. Conti, M. Guerra, D. Dardari, N. Decarli, and M. Z. Win, "Network experimentation for cooperative localization," IEEE J. Select. Areas Commun., vol. 30, no. 2, pp. 467-475, Feb. 2012.

[10] D. Dardari and R. D'Errico, "Passive ultrawide bandwidth RFID," in IEEE Global Communications Conference, New Orelans, LA, USA, Nov. 2008.

[11] V. Heiries, K. Belmkaddem, F. Dehmas, B. Denis, L. Ouvry, and R. D'Errico, "UWB backscattering system for passive RFID tag ranging and tracking," in 2011 IEEE International Conference on UltraWideband, pp. 489-493, Sep. 2011.

[12] C. Xu and C. L. Law, "TOA estimator for UWB backscattering RFID system with clutter suppression capability," EURASIP Journal on Wireless Communications and Networking, 2010.

[13] D. Dardari, F. Guidi, C. Roblin, and A. Sibille, "Ultra-wide bandwidth backscatter modulation: Processing schemes and performance," EURASIP Journal on Wireless Communications and Networking, vol. 2011, no. 1, 2011.

[14] B. Long, P. Zhang, and J. Hu, "A generalized QS-CDMA system and the design of new spreading codes," IEEE Transactions on Vehicular Technology, vol. 47, no. 4, pp. 1268-1275, Nov. 1998.

[15] P. Fan, "Spreading sequence design and theoretical limits for quasisynchronous CDMA systems," EURASIP Journal on Wireless Communications and Networking, vol. 2004, no. 1, 2004.

[16] A. Goldsmith, Wireless Communications Cambridge University Press, New York, 2005.

[17] H. Donelan and T. O'Farrell, "Method for generating sets of orthogonal sequences," IEEE Electronics Letters, vol. 35, no. 18, pp. 1537-1538, Sep. 1999.

[18] R. De Gaudenzi, C. Elia, and R. Viola, "Bandlimited quasi-synchronous CDMA: a novel satellite access technique for mobile and personal communication systems," IEEE Journal on Selected Areas in Communications, vol. 10, no. 2, pp. 328-343, Feb. 1992. 